\title{
Viejos/Nuevos enemigos en América del Sur: revisitando el concepto de hostilidad
}

Ivan WITKER*

El problema más serio que implica el control democrático de la politica exterior es que ningún gobierno puede permitirse divulgar con franqueza toda la información sobre su fortaleza militar y su conocimiento sobre la fortaleza de los otros países.

E.H. CARR ${ }^{1}$

$\mathrm{E}$ n el híbrido teórico de internacionalistas y cientistas políticos hay consenso en torno a que se vive un profundo tránsito conceptual en los esfuerzos por tratar de comprender las nuevas configuraciones de poder internacional. Este momento transicional se torna elocuente cuando, desde las relaciones internacionales, se hace una aproximación a asuntos específicos de Seguridad y Defensa.

Por ello, uno de los ejes de dicho tránsito radica en la díada Guerra/Paz. Y es que acabada la pesadilla de una conflagración nuclear en la década del 90, se propagó una idea matriz de las corrientes idealistas en orden a que los conflictos dejarían paulatinamente de tener un cariz violento y guerrero, para estar marcados por un afán resolutivo de tipo cooperativo y pacífico, teniendo como bases el derecho internacional, la fuerza coercitiva de la opinión pública internacional, pero ante todo la arquitectura multilateral supranacional. Ergo, en lugar de tener un trasfondo polemo-

\footnotetext{
* PhD. por la Universidad Carlos IV de Praga. Actualmente es Jefe de la Cátedra de Relaciones Internacionales de la Academia Nacional de Estudios Políticos y Estratégicos, ANEPE, y profesor-investigador del Instituto de Estudios Avanzados, USACH. Correo electrónico: ivanwitker@yahoo.com

1. La crisis de los veinte años, Cataratas, Madrid, 2004, p. 146.

Nota: Este artículo está vinculado al proyecto Fondecyt $N^{\circ} 1030017$ Estado, Sociedad y Terrorismo, básicamente en lo referido al debate conceptual. Una versión preliminar fue presentada en el 7º Congreso de Ciencia Política, Santiago, agosto, 2004.
}

Política. Volumen 44 - Otoño 2005, pp. 247-256 
lógico, o un determinismo polemológico, las relaciones internacionales pasarían a tener uno signado por la irenología ${ }^{2}$.

Mas los atentados terroristas de Nueva York y Washington (2001) y Madrid (2003) no han hecho otra cosa que recolocar el tema de la violencia política en el centro del debate y cuestionar la comitas gentium con que se estaban abordando las cuestiones internacionales.

Ese hiato denominado post-Guerra Fría concluyó abruptamente con estos dramáticos hechos, dando paso a una focalización teórica intensa en el concepto conflicto. Esta noción intermedia entre Guerra y Paz ha devenido en un eje del debate epistemológico actual.

En el plano local, el Segundo Libro de la Defensa Nacional de Chile sostiene que el conflicto contemporáneo se desglosa en crisis (su forma más recurrente) y guerra. En tanto, para Charles Doran (2003), uno de los principales especialistas en conflictos internacionales, resulta fundamental, a la hora de reflexionar sobre la díada Guerra/Paz y sus categorías intermedias, modificar la pregunta rectora tradicional, del porqué las naciones van a la guerra, por la de cuándo las naciones deciden ir a una guerra. La respuesta nos permite, a su juicio, diferenciar los matices e intensidades del conflicto internacional ${ }^{3}$.

Ahora bien, si focalizamos la atención hacia las peculiaridades de los conflictos en América del Sur, parece pertinente explorar los alcances de una noción que podría servir de instrumento para explicar algunas conductas externas de los Estados de la región y de las nuevas configuraciones estratégicas que se perfilan en el plano regional: la hostilidad.

El concepto no es nuevo en la ciencia política. Lo usó seminalmente Maquiavelo en varias de sus obras centrales (Arte de la Guerra, Misiones e Historia de Florencia) para explicar el intrincado juego de alianzas y confrontaciones entre los minúsculos Estados-ciudades de la Italia renacen-

2. Algunos autores hablan de la tendencia hacia la "civilización o domesticación de los conflictos interestatales". Esta tendencia se produciría debido al espanto que causa en el individuo moderno la cantidad de víctimas registradas durante el siglo XX durante las dos guerras mundiales, y los innumerables conflictos armados, guerras, golpes de Estado, guerrillas y contraguerrillas, guerras de baja intensidad, revoluciones, guerras civiles y guerras de liberación. Productos de esta tendencia serían las múltiples técnicas de resolución y gestión de conflictos surgidas estas últimas décadas. Por lo mismo, la base de los estudios sobre la paz y y sus pemisas (la irenología) es que la paz es más que el simple estado de no-guerra, o ausencia de violencia, en lenguaje de Galtung (Woyke, 2002).

3. En el plano regional, es fundamental la obra Boundary Disputes in Latin America, editada por Jorge Domínguez y publicada por el United States Institute of Peace, 2003 (comp.), donde se analizan los diferendos territoriales, contenciosos limítrofes y disputas militares, todo ello en función de su posible escalamiento o contención poniéndolos en perspectiva kantiana, o wilsoniana si se prefiere, y examinan sobre cuánto contribuiría la democracia a su posible solución. Todos subrayan la excepcionalidad de la región en lo que respecta al bajo número de guerras, así como de conflictos armados de intensidad reducida. Entre las claves para entender dicha excepcionalidad, mencionan: el éxito de la doctrina del uti possideti en el siglo XIX, con sólo 6 excepciones, y el de la disuasión en el siglo XX con sólo dos excepciones (Perú/Ecuador, Paraguay/Bolivia). Esta obra permite -retomando la percepción de Maquiavelo- diferenciar el concepto de conflicto del de hostilidad. 
tista, entendiéndolo como un estado de cosas previo al conflicto propiamente tal.

En la actualidad se considera interesante el aporte metodológico de Nils Jordet de la Fletcher School of Law and Diplomacy de la Tufts University, para quien la hostilidad debe, necesariamente, definirse en función de su temporalidad, o sea, si se trata de una situación de largo o de corto plazo, así como de los niveles de animosidad de uno en contra de otro; ésta debe observarse, dice Jordet, en cuatro ámbitos: el político, el diplomático, el militar y el económico (Jordet, 2001). Distingue tres aproximaciones analíticas a un hecho de hostilidad. Una de carácter descriptivo, orientada a clasificar, medir y comparar los componentes del hecho hostil estudiado; otra aproximación de tipo causa-efecto, que busca establecer relaciones entre los cuatro ámbitos descritos o en una cadena de hechos hostiles; y, finalmente, una aproximación de emocionalidad (emotional issues), que examina las confianzas y los tipos de percepción entre los involucrados ${ }^{4}$.

Siguiendo la metodología de Jordet, podemos avanzar tres hipótesis: que la hostilidad es un concepto diferenciable del conflicto; que la hostilidad no es influenciable por la uniformidad de regímenes políticos entre los involucrados; que el nudo desde donde ramifican los sentimientos de hostilidad se encuentra en el ámbito de las emocionalidades. Asimismo, teniendo como guía el dictum de Waltz, en orden a que explicar una guerra es más fácil que entender las condiciones para la paz, nos parece plausible comprender la hostilidad como una derivada del estado de anarquía en que vive el sistema internacional (Waltz, 1979: 204). Hostilidad es, entonces, una conducta inamistosa acotada, específica, visible en tiempos de paz, de carácter público e intuida como tal ${ }^{5}$. Se

4. Otros autores referenciales son:

- Brzezinski, Zbigniew (2004) Hostility to America has never been so great, New Perspectives Quarterly, vol. 21, $\mathrm{N}^{\circ} 3$ summer, donde le otorga el rango de criterio central para definir el clima internacional que envuelve a un Estado determinado;

- Davis, Davies, G. (2002) Diversionary conflict. An empirical test 1950-1982, Manchester Papers in Politics CIP Series, Manchester, y Boehmer, Charles (2002) Domestic crisis and interstate conflict: the impact of economic crisis, domestic discord and state efficacy on the decision to initiate interstate conflict. Ponencia presentada en el Congreso de la Asociación de Estudios Internacionales, marzo, 24, en Nueva Orleans. Ambos asumen la hostilidad como componente de los los llamados conflictos distractivos (diversionary conflicts);

- Hensel, Paul (1999) An evolutionary approach to the study of interstate rivalry, Conflict Management and Peace Science 17 (2), otoño, quien analiza el concepto en el contexto de las rivalidades interestatales.

- Leyton, Cristián (2001) El factor de amenaza: ejes de la percepción de amenaza chilena Revista CIDOB d'Afers internacionals $\mathrm{N}^{\circ}$ 51-52, Barcelona, enero, quien lo sitúa exclusivamente en el plano interestatal, de ahí la conexión directa que establece con las nociones de amenaza y percepción de amenaza teniendo los conceptos de configuración territorial y equilibrio militar regional como trasfondo explicativo.

5. Intuida en términos kantianos. En la filosofía ha habido un largo debate acerca de la diferenciación entre sensación y percepción. Para Kant, esta última está unida a la idea de experimentar, de ser percibida como tal en virtud de un conocimiento previo. Algunos filósofos hablan del "contenido no conceptual" de una experiencia. Esta sutileza no es menor para efectos de esta reflexión, toda vez que un acto de hostilidad debe ser "intuido" como tal por la parte hostilizada. 
trata de un acto que por sí mismo no amenaza la estabilidad del sistema internacional, o de una región en particular, pero su recurrencia deteriora el clima pues puede provocar contrarreacciones a nivel estatal y escalar a un conflicto. En tal sentido, percibimos el concepto de hostilidad en el sentido schmittiano, es decir, proveniente de un adversario público, visible, no de un inimicus, sino de un hostis (Schmitt, 1996 [1928]).

Esta propuesta de definición nos permite ir a la raíz de las diferencias entre Conflicto y Hostilidad, y avanzar, por esta vía, hacia una reflexión más atingente a la realidad sudamericana. Así entonces, en el primero observamos la concurrencia de dos o más Estados, mientras el segundo exhibe un carácter unilateral. El primero depende, en sus partes y su totalidad, de la acción de la autoridad, pudiendo ésta atenuarlos o eliminarlos mediante negociaciones, o bien dejarlo en estado latente si no consigue llevar a buen puerto las negociaciones o se niega a éstas. La hostilidad en cambio no tiene relación necesariamente con las estructuras centrales del Estado, sino más bien son actitudes, conductas y gestos, que se dan de manera dispersa, muchas veces de forma inorgánica o paraorgánica, pues en su generación intervienen también actores transnacionales, actores no estatales, actores subnacionales e incluso últimamente los medios de comunicación, que alientan sentimientos hostiles al generar o diseminar de manera caótica opiniones y valoraciones sobre conductas de otras naciones. Esta dispersión de fuentes generadoras de un hecho hostil se observa de manera nítida en nuestra región, producto de una comprensión primaria de la democracia, mimetizada con meros ejercicios electorales y con un marco de garantía de ciertas libertades personales o actorales. Esta dispersión de fuentes generadoras provoca actos hostiles muchas veces a partir de coyunturas sumamente triviales.

La teoría de las relaciones internacionales ilustra este problema con un ejemplo latinoamericano, el de la Guerra del Fútbol entre Honduras y El Salvador en 1969; allí se observa el ciclo completo: de una sucesión de actos y sentimientos hostiles relativamente aislados, se escala a una abierta hostilidad interestatal, y rápidamente a conflicto manejado sin pericia por las respectivas elites políticas, desencadenándose una guerra ${ }^{6}$.

Este ejemplo nos ilustra en el sentido de que hay momentos en que el Estado y estamentos de la Sociedad convergen para crear un peligroso tipo de hostilidad interestatal, que debe ser entendido en el contexto de las amenazas reales o potenciales que se ciernen sobre un determinado país y que representan una etapa superior de la hostilidad. El ciclo completo de esta relación ascendiente está formado por las nociones de Hostilidad > Hostilidad Interestatal (presencia de amenazas reales o potenciales) $>$ Conflicto $>$ Guerra.

Por lo tanto, cuando una cadena de actos hostiles tiene como fuente generadora al Estado y a la Sociedad por igual, éstos pueden ser percibi-

6. Esta guerra (julio, 1969) duró sólo cinco días, pero dejó sobre 3 mil muertos; concluyó gracias a un cese al fuego patøcinado por la OEA. 
dos por el otro como signos que presagian peligros reales. Entonces, estamos en presencia de antagonismos, y de una posible colisión de intereses, accediendo al umbral del conflicto. Por esta razón, al estudio de la hostilidad pertenecen actividades vitales para un Estado como son la identificación de intenciones y evaluación de capacidades del adversario potencial, el aislamiento de señales, el desciframiento de ruidos al interior de toda relación bilateral y el examen permanente del clima que rodea la presencia internacional del Estado.

En consecuencia, al calor de la transición conceptual mencionada $u t s u$ pra, parece pertinente interrogarse acerca del impacto que tiene (o puede llegar a tener) la democracia sobre las situaciones descritas. Según se desprende de la obra compilada por Domínguez, los conflictos históricos en esta región del planeta $-y$ por ende las hostilidades- mantienen una latencia parecida a la de antaño, y la influencia directa de la democracia o de la apertura comercial -también mencionada por algunos autores como instrumento coactivo- es relativamente baja. Ciertamente que explicaciones importantes radican en: el carácter "imperfecto" de las democracias sudamericanas, en el decir de O'Donnell, en el hecho que en los países sudamericanos se registraron reformas políticas y económicas más bien aisladas, sin un adecuado respaldo jurídico-legal ni bases institucionales, y, obviamente, en reminiscencias de animadversión histórica. Por lo tanto, si las cosas funcionan en una parte y en la otra sólo se aprecian fenómenos anómicos, es comprensible que nazcan o reverdezcan manifestaciones hostiles hacia el primero.

La experiencia internacional indica que si la democracia es entendida y asumida de forma compleja y responsable, institucional y madura (en el sentido kantiano de Rechtsstaat), y de manera simultánea por varios países circunvecinos, las fuentes generadoras de hostilidad debieran constreñirse. Esa es la lección de la convergencia europea. Los exitosos esfuerzos de Jean Monnet y Robert Schuman después de la Segunda Guerra Mundial se orientaron justamente a transformar la democracia en un compromiso ciudadano maduro, plasmable en cuestiones concretas y mutuamente beneficiosas para Francia y Alemania, como método de cooperación que contribuyese a finalizar guerras, conflictos y hostilidades centenarias.

La disparidad observable en América del Sur en materia de madurez democrática unida a la perceptible rivalidad estatal coadyuvada por la pervivencia de animosidades históricas sirven para ilustrar los desafíos que tiene Chile ante sí en materia de hostilidades.

El país ciertamente no vive situaciones de conflicto con algunos de los vecinos; tampoco con alguno de los países del resto de la región sudamericana. Sí, en cambio, existen ruidos y señales con ciertas intermitencias, susceptibles de ser interpretadas como actos de hostilidad de parte de varios vecinos y algunos paravecinos. En los últimos años, sólo gracias a un cauto manejo de la Administración Lagos no se ha derivado a un estado de hostilidad interestatal. 
En efecto, la rivalidad estatal ha adquirido fuerza básicamente por el hecho de que Chile logró identificar sus imperativos estratégicos, tras casi diez años de dificultades en dicha tarea debido a las complejidades internas de la transición y a que tomó tiempo para que se consolide la idea de que las instituciones armadas no son entidades ajenas al quehacer nacional. Tal identificación es el mejor ejemplo que la democracia se asentó y maduró.

En efecto, por un lado, con el ingreso a la APEC y con la posterior suscripción de Tratados de Libre Comercio con EE.UU. y la Unión Europea, se afianzó el establecimiento de prioridades políticas y económicas en el trabajo externo de Chile. Por otra parte, se logró conceptualmente a través del Segundo Libro de la Defensa Nacional, plasmar una visión más nítida respecto a la región, perfilando tres nociones de mayor relevancia para esta renovada presencia internacional del país: la cooperación, el uso coercitivo de la fuerza y la disuasión. La identificación de imperativos estratégicos es desde el punto de vista del Estado de Chile un logro histórico, obtenido sobre la base de un consenso político y sectorial bastante razonable, único en el plano regional. Esta realidad transforma al país en una excepcionalidad, pues en prácticamente todos los países de nuestro entorno, observamos un enfrentamiento político interno que paraliza un diseño de ejecución de política exterior consensuada y predecible. En tal sentido, esta identificación de imperativos estratégicos por parte de Chile es motivo de rivalidad, tan sólo por gozar de tan amplio sustento y base de legitimidad ${ }^{7}$. Aún más, la decisión de intervenir en Haití bajo el formato de Imposición de la Paz junto a grandes potencias, inició un paulatino y seguro acercamiento de las políticas Exterior y de Defensa, lo que ha permitido darle consistencia a esta identificación de imperativos, y, de paso, elevar la estatura estratégica del país ${ }^{8}$.

El tema de la rivalidad no es enteramente nuevo, aunque sí está asumiendo un cariz especial estos últimos años. Por ejemplo, el ingreso a la APEC, como único país sudamericano, generó una situación de antagonismo de tipo estático con varios países de la región, que aspiraban a dar un paso parecido en ese mismo momento o pocos años más tarde; situación improbable toda vez que el poder decisorio efectivo en ese conglomerado no lo tienen los latinoamericanos, de ahí el carácter estático. Por otro lado, la opción chilena de priorizar el acercamiento con el mundo desarrollado, y la reacción de éste, de preferir negociar con un país intermedio como Chile antes que con otros que históricamente han tenido

7. La rivalidad en este caso debe entenderse como antagonismo. La identificación de imperativos estratégicos, lo que es percibido por los países mencionados como conducta y discurso poco solidarios con los infortunios latinoamericanos en general y como excesivamente funcional a la politica exterior estadounidense.

8. Este es un concepto de uso reciente en las relaciones internacionales. El Dr. Rafael Calduch (1996) lo define como "la aptitud de un Estado para proyectar su poder, para avalar sus decisiones internacionales o su voluntad política en un ámbito específico de relaciones de poder". Dada la naturaleza asimétrica de las relaciones de poder en el contexto internacional, la estatura estratégica de un Estado está en directa relación con sus capacidades y aspiraciones. 
una mayor carga de tipo simbólico-representativo de esta región del mundo, ha generado un antagonismo, esta vez de tipo activo, toda vez que forma parte de las nuevas correlaciones de fuerza en el plano regional y de las prioridades que se ha fijado cada país sudamericano. Parte de esta rivalidad respecto al mundo desarrollado se atisba también en los críticos discursos del presidente Chávez sobre el ALCA y del Presidente Kirchner sobre las instituciones financieras internacionales diametralmente opuestas en forma y contenido a la política exterior de Chile. Luego, otro componente de este antagonismo activo es el hecho que la satisfacción de la población chilena con la economía de mercado es excepcionalmente alta en la región, según lo demuestra la última encuesta de Latinobarómetro, lo que coadyuva a ver los gestos y los discursos de Chávez y de Kirchner con distancia y con recelo.

Ahora bien, si a este cuadro se une un cierto descuido estratégico en el trato político del Chile democrático hacia su entorno vecinal y paravecinal, y un cierto descanso en los aspectos meramente formales de la relación bilateral, se pueden entender de mejor manera los actos de hostilidad apreciables en los últimos años respecto a Chile. Y es que la relación con los vecinos, especialmente cuando se atraviesa por un período de excepcionalidad, requiere una permanente proactividad, que, en este caso, pasa por cambiar de posición en el contexto de las asimetrías propias de toda relación internacional.

En efecto, Chile, casi por acto reflejo, ha asumido históricamente el lado débil y pasivo en cualquier relación, situación que evidentemente colisiona hoy día con los datos duros de la realidad regional, como son el crecimiento económico sostenido, la fortaleza institucional, calidad de la democracia, la predictibilidad internacional y el grado de interlocución alcanzado con las potencias centrales por parte de Chile. Se observa que Chile ha hecho caso omiso de un asunto esencial de las relaciones internacionales, que actuar en la parte fuerte de una relación asimétrica es por norma más difícil, que suele tener costos importantes, y que es inevitable cuando estos datos duros forman parte esencial de los flujos informativos en una societas eruditorum, o societas videns en el decir de Sartori. Asimismo, la necesaria proactividad respecto al ámbito vecinal y paravecinal, exigía estar atento a la circunstancia que las opciones de desarrollo visualizadas en América del Sur son las determinantes de las nuevas configuraciones estratégicas en la región. No es casual que Venezuela y Argentina -países con evidente falencias y rezagos en las materias diferenciadoras señaladas ut supra- sean dos países desde donde provienen importantes gestos de hostilidad hacia Chile. Tampoco es casual que entre Caracas y Buenos Aires se haya gestado un eje estratégico con el que Chile poco o nada puede compartir.

En tanto, la pervivencia de animadversiones de tipo histórico se remite al hecho que el Imperio español en América fue una suerte de archipiélago formado por islas de hispanización en medio de un océano indígena de etnias y lenguas diversas. Dichas islas hispanizadas de los virreinatos 
reunían todo lo que en Europa se requería para definir una nacionalidad: lengua, religión, cultura, historia y, en medida variable según el grado de mestización, también raza. Con el colapso de la Metrópoli, el archipiélago hispanizado se desmembró en soberanías varias, ya que ningún centro hispanoamericano tenía poder suficiente para mantener unificado un territorio tan vasto. Por ello, la falta de diferenciación en los Estados embrionarios representó un primer grave problema de gobernabilidad. Ergo, la identidad y la fijación de fronteras debieron crearse y materializarse a partir de una agudización de la diferenciación, produciendo heterogeneidad a través de la creación de estereotipos y de un adoctrinamiento nacionalista de muy largo plazo sobre el carácter codicioso, desleal, violento y expansionista del vecino. Un proceso diferenciador muy distinto al seguido por Brasil, país que ya al finalizar el siglo XIX exhibe el inédito logro de haber fijado sus fronteras mediante tratativas pacíficas bilaterales o por medio de arbitrajes con cada uno de sus múltiples vecinos gracias a la habilidad negociadora del barón de Río Branco, lo que reforzó y aceleró la maduración de un proceso identitario muy distinto al de las ex colonias españolas ${ }^{9}$. Los mecanismos privilegiados en la América hispana, por el contrario, se consolidaron a través de un sistema educativo ad hoc a la generación de ruidos hostiles cuando no de conflictos e incluso guerras. Estas variables históricas se encuentran tan afincadas en el subconsciente de algunos pueblos sudamericanos que revertirlas hoy es una tarea compleja y larga, e imposible si no existe voluntad política expresa.

Chile, pese a no ser ajeno a esta tendencia, exhibe rasgos diferenciadores importantes. En efecto, gran parte del ethos de sus Fuerzas Armadas radica precisamente en que éstas fueron capaces de superar las percepciones de inseguridad que corroían a la nación chilena en sus primeros años de formación, consiguiendo erigirse en fundadores de la nueva nación, no sólo liberadores del poder colonial, como ocurre en los demás países de la región. La especial relación de las FF.AA. con la civilidad y con el poder político a lo largo de los dos siglos de historia republicana, destacando obviamente la experiencia 73-89 y las características de la transición de terciopelo vivida en los 90, tiene un distintivo muy claro en la región, estableciendo una gama de singularidades.

En una somera mirada a cuestiones empíricas, destaca que las percepciones de hostilidad desde Chile han cobrado nuevos bríos. Y es que, pese a que ya ha conseguido vivir 120 años en paz, pese a que su estatura estratégica ha aumentado considerablemente, y pese a que su propio status dentro de los países en vías de desarrollo ha mejorado sustantivamente, la sensación de vulnerabilidad, y de tener un entorno hostil, per-

9. La envergadura diplomática de Río Branco, como escribe Celso Lafer (2002: 55), es indiscutible. Es difícil encontrar en la historia de las relaciones internacionales un desempeño negociador y un patrón exclusivamente pacífico semejante a los brasileños en la fijación de sus fronteras nacionales. Este desempeño se caracterizó por la concentración metódica, sistemática, de todos los recursos diplomáticos y del uso legítimo, no violento, del poder, sin llegar al conflicto militar, para la solución con éxito del conjunto de problemas fronterizos. 
siste. Hoy son dos las grandes preocupaciones: la escasa profundidad estratégica de su territorio, principalmente horizontal, y en sus problemas para asegurar abastecimiento energético ${ }^{10}$.

Es en este último punto donde los chilenos han percibido más recientemente acciones de vecinos que claramente llevan el signo de hostilidad; amén de que antaño pudiesen haber sido percibidas directamente como agresiones. Elocuente es el Acuerdo de Exportación de Gas recientemente firmado por Bolivia y Argentina, donde quedó consignada la famosa frase "ni una sola molécula hacia Chile". Debe recordarse que no sólo se trata de dos países vecinos, con uno de los cuáles Chile nunca ha tenido guerra y con el cual estaba concluyendo una de las décadas más productivas de la historia en materia de colaboración bilateral, sino que con ambos comparte membresía en Mercosur. Lo ocurrido con el suministro energético golpeó, pues el respeto por los tratados se encuentra profundamente arraigado en el imaginario e idiosincrasia de los chilenos, un histórico y marcado elemento diferenciador con el resto de la región ${ }^{11}$.

En síntesis, de esta revisita al concepto de hostilidad se desprende que se le debe entender como acto acotado ${ }^{12}$, cuyo encadenamiento se puede revertir hasta cierto límite mediante contra-gestos y mediante un espíritu maduro que no cierra el paso al ánimo cooperativo. Ello queda corroborado en el hecho de que, pese a los ruidos y señales de hostilidad del entorno, el país ha sido capaz de tales contra-gestos. El intento peruano, por ejemplo, de renegociar la delimitación marítima, amenazando con llevar el caso al Tribunal de La Haya, fue replicado con ese elocuente contra-gesto del propio Comandante en Jefe del Ejército de Chile de invitar a su homólogo peruano a conmemorar juntos una efeméride nacional de relevancia (agosto, 2004).

La experiencia internacional indica que las naciones que han alcanzado definiciones de carácter estratégico, i.e. un nivel maduro de desarrollo democrático y han definido un modelo de desarrollo económico para el largo plazo, están obligadas a convivir con manifestaciones hostiles cuando su vecindario permanece en un status disfuncional en ambas definiciones. Por lo tanto, para abordar adecuadamente los desafíos que emanan de una realidad heterogénea, las claves radican en dotar al Esta-

10. Por profundidad estratégica se entiende en relaciones internacionales la distancia que separa los núcleos vitales de los límites estatales.

11. La llamada clausula rebus sic stantibus y el principio de pacta sunt servanda pertenecen a lo más íntimo de la visión chilena de los asuntos internacionales. El problema suscitado con los argentinos y bolivianos tiene que ver más bien con el neoidealismo imperante en los círculos decisorios chilenos. La historia de las relaciones internacionales registra el caso de Francia en 1848, cuando oficialmente declaró de forma unilateral que "los tratados de 1815 ya no son válidos a los ojos de la República Francesa", como el primer caso de una potencia que deja de otorgarle validez a un documento de esta maturaleza.

12. Un excelente ejemplo de ello es la conducta del presidente venezolano Hugo Chávez respecto a Chile en el bienio 2004-2005. Pese a haber generado intermitencias de hostilidad, a propósito de la demanda boliviana de salida al mar, cerró el período citado como un importante promotor de la candidatura de José Miguel Insulza a la Secretaría General de la Organización de Estados Americanos (OEA). 
do de instrumentos y recursos que permitan materializar aquellos tres conceptos rectores contenidos en el Segundo Libro de la Defensa, la disuasión, el uso coercitivo de la fuerza y la cooperación.

\section{Bibliografía}

Calduch, Rafael. 1996. Conferencia Los desafíos de la paz en la ex Unión Soviética, Madrid, junio de 1996, texto en Biblioteca del Centro de Estudios de la Defensa Nacional (CESEDEN). Citado por Contreras Polgatti, Arturo en su tesis doctoral, Instituto de Estudios Avanzados, Universidad de Santiago, septiembre, 2004.

Doran, Charles. 1 de julio de 2003. Power circle theory, absolute and relative gains and the strategic environment at critical point, ponencia en el Congreso Mundial de la Asociación Internacional de Ciencia Política, Durban, Sudáfrica.

Jordet, Nils. 2001. Explaining the long-term Hostility between the USA and Iran: a historical, theoretical and methodological framework, (paper) Tufts University, Boston.

Lafer, Celso. 2002. La identidad internacional de Brasil, FCE, México.

Schmitt, Carl. 1996 [1928]. The Concept of the Political, The University of Chicago Press, Chicago.

Waltz, Kenneth. 1979. Theory of international politics. McGraw-Hill, New York.

Woyke, W. 2002. Handwörterbuch internationale Politik, Leske-Budrich, Stuttgart, (Krieg und Frieden): 241-261. 